\title{
Yap haploinsufficiency leads to Müller cell dysfunction and late-onset cone dystrophy
}

\author{
Christel Masson', Diana García-García', Juliette Bitard ${ }^{1}$, Élodie-Kim Grellier ${ }^{1}$, Jérôme E. Roger ${ }^{1}$ and Muriel Perron (1)
}

\begin{abstract}
Hippo signalling regulates eye growth during embryogenesis through its effectors YAP and TAZ. Taking advantage of a Yap heterozygous mouse line, we here sought to examine its function in adult neural retina, where YAP expression is restricted to Müller glia. We first discovered an unexpected temporal dynamic of gene compensation. At postnatal stages, Taz upregulation occurs, leading to a gain of function-like phenotype characterised by EGFR signalling potentiation and delayed cell-cycle exit of retinal progenitors. In contrast, $\mathrm{Yap}^{+/-}$adult retinas no longer exhibit TAZdependent dosage compensation. In this context, Yap haploinsufficiency in aged individuals results in Müller glia dysfunction, late-onset cone degeneration, and reduced cone-mediated visual response. Alteration of glial homeostasis and altered patterns of cone opsins were also observed in Müller cell-specific conditional Yap-knockout aged mice. Together, this study highlights a novel YAP function in Müller cells for the maintenance of retinal tissue homeostasis and the preservation of cone integrity. It also suggests that YAP haploinsufficiency should be considered and explored as a cause of cone dystrophies in human.
\end{abstract}

\section{Introduction}

Deregulation of key signalling pathways, such as Wnt or Notch, well known for their involvement in retinal development, are at the origin of retinal diseases in adults ${ }^{1,2}$. Studying developmental signalling pathways can thus allow the identification of possible causes of retinal disorders in adults, pinpointing possible targets for therapeutic approaches. The Hippo signalling tightly regulates eye development, and its deregulation in animal models leads to severe ocular defects (reviewed $\mathrm{in}^{3,4}$ ). This signalling pathway consists of a kinase cascade that ultimately phosphorylates the transcription coactivators YAP (Yes associated protein) and TAZ (WW domain-containing transcription regulator 1 ), causing their retention in the cytoplasm or their degradation. When the pathway is inactive, YAP/TAZ are translocated to the nucleus, leading

\footnotetext{
Correspondence: Christel Masson (christel.masson-garcia@universite-parissaclay.fr) or Jérôme E. Roger (jerome.roger@universite-paris-saclay.fr) or Muriel Perron (muriel.perron@universite-paris-saclay.fr)

${ }^{1}$ Paris-Saclay Institute of Neuroscience, CERTO-Retina France, CNRS, Université Paris-Saclay, Orsay 91405, France
}

Edited by S. Inoue to activation of their target genes ${ }^{5,6}$. During retinal development, YAP and TAZ are expressed in all optic vesicle compartments, becoming more prominently expressed in the retinal pigment epithelium (RPE) and the ciliary margin of the optic cup ${ }^{4,7}$. Studies in zebrafish and mice indicate that YAP is necessary for the maintenance of progenitor populations in both the retina and the $\mathrm{RPE}^{4,7-11}$. Besides, mutations in Hippo pathway components have been identified in human ocular diseases, such as the Sveinsson's chorioretinal atrophy (SCRA) ${ }^{12,13}$, in patients with ocular manifestations of the neurofibromatosis type-2 human disease $^{14}$, or in patients exhibiting coloboma ${ }^{15,16}$. Together, these studies have contributed to highlight the important role of the Hippo-YAP pathway in eye development and ocular disorders. Although it has previously been established that YAP remains expressed in the adult retina in both Müller glia and RPE cells ${ }^{17}$, its function in the adult eye has received little attention thus far.

To investigate YAP function in the adult, and because Yap knockout is lethal at embryonic day $8.5^{18}$, we took advantage of a Yap heterozygous mouse model. We found that depletion of one Yap allele leads to a transient TAZ 
compensatory mechanism at postnatal stages, associated with an increased expression of YAP/TAZ target genes. Consistent with the known critical role of the Hippo pathway in regulating neural progenitor cell proliferation $^{19}$, we found that such enhanced TAZ activity in $\mathrm{Yap}^{+/-}$postnatal retinas is associated with a gain of function-like phenotype, i.e., delayed cell-cycle exit of retinal progenitors. We identified the regulation of the EGFR (Epidermal Growth Factor Receptor) pathway as a potential underlying mechanism. Conversely, TAZ compensatory regulation declined during aging in Yap heterozygous retinas. We found that non-compensated decrease of Yap expression in aged retinas leads to altered Müller cell homeostatic function, causing late-onset cone degeneration and thereby impaired cone visual function. This work thus uncovers a novel unexpected role for YAP in cone photoreceptor maintenance and proper vision.

\section{Methods and material Ethics statement}

All animal experiments have been carried out in accordance with the European Communities Council Directive of 22 September 2010 (2010/63/EEC). All animal care and experimentation were conducted in accordance with institutional guidelines, under the institutional license D 91-272-105. The study protocols were approved by the institutional animal care committee CEEA $\mathrm{n}^{\circ} 59$ and received an authorisation by the "Direction Départementale de la Protection des Populations" under the reference APAFIS\#1018-2016072611404304 v1.

\section{Mice}

Mice were kept at $21^{\circ} \mathrm{C}$, under a 12-h light/12-h dark cycle, with food and water supplied ad libitum. Heterozygous $\mathrm{Yap}^{+/-}$mice were obtained from Sigolene Meilhac (Institut Pasteur, Paris). Briefly, Yap ${ }^{f l o x /+}$ mice $^{20}$ were crossed with PKG Cre mice (PGK-Cre transgene is maternally expressed and serves as a tool for early and uniform activation of the Cre site-specific recombinase ${ }^{21}$ ) to generate the $\mathrm{Yap}^{+/-}$mice, that are viable and fertile. Yap $^{\text {flox/flox }}$;Rax-CreER ${ }^{T 2}$ mice were obtained as previously

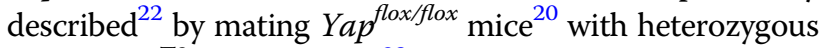
Rax-CreER ${ }^{\mathrm{T} 2}$ knockin mice ${ }^{23}$. The Cre activity was induced through a single intraperitoneal injection of 4-hydroxytamoxifen (4-OHT; $1 \mathrm{mg} / \mathrm{kg}$ ) at P10. Genotyping was done by PCR using genomic DNA prepared from mouse tail snips. Primers are provided in Supplementary Table S1.

\section{Tissue sectioning, immunohistochemistry, and EdU labelling}

Eyes of sacrificed animals were rapidly enucleated and dissected in Hanks' Balanced Salt solution (Gibco) to obtain posterior segment eye-cups, which were then fixed in $4 \%$ paraformaldehyde, $1 \mathrm{X}$ PBS, for $1 \mathrm{~h}$ at $4{ }^{\circ} \mathrm{C}$. Eye-cups were then dehydrated, embedded in paraffin and sectioned $(7 \mu \mathrm{m})$ with a Microm HM 340E microtome (Thermo Scientific). Hematoxylin-eosin (H\&E) staining was performed according to the manufacturer's instructions (BBC Biochemical). Stained sections were mounted with Eukitt (Polylabo). Standard immunohistochemistry techniques on paraffin sections were applied with the following specificities: antigen unmasking treatment was done in boiling heat-mediated antigen retrieval buffer (10 mM sodium citrate, $\mathrm{pH}$ 6.0) for $20 \mathrm{~min}$. Primary antibody was diluted in ready-to-use diluent (Dako). Primary and secondary antibodies are listed in Supplementary Table S2. Sections were counterstained with $1 \mu \mathrm{g} / \mathrm{ml}$ DAPI (Thermo Fisher Scientific) and mounted with Fluor Save $^{\mathrm{TM}}$ reagent (Millipore). For retinal flat mounts, small dorsal incisions were made before fixation to mark the orientation of the retina within the eyecup. For EdU incorporation experiments, mice were given a single 50-100 $\mu \mathrm{l}$ intraperitoneal injection of $10 \mu \mathrm{M}$ of EdU (Invitrogen) at the indicated stage. EdU incorporation was detected on flat-mounted retinal mouse explants using the Click-iT EdU Imaging Kit (Invitrogen) according to manufacturer's recommendations. For double labelling, EdU labelling was done first, followed by immunostaining.

\section{Imaging}

Fluorescence images were acquired using a LSM710 confocal microscope (Zeiss). Whole retina images were acquired using an AXIOZoom.V16 (Zeiss) using the mosaic mode. Image mosaics of flat-mounted retinas were acquired and combined by the stitching processing method using ZEN Tiles module (Zeiss). Brightfield images of H\&E staining were acquired using an AxioImager.M2 microscope. Image processing was performed using Zen 2.1 (Zeiss), Fiji (National Institutes of Health ${ }^{24}$ ), and Photoshop CS4 software (Adobe) software. The same magnification, laser intensity, gain, and offset settings were used across animals for any given marker.

\section{Western blotting}

Western blot was performed on protein extracts from single retinas, at least on three individuals per condition, unless otherwise specified in the figure legends. Retinas from enucleated eyes were dissected in Hanks' Balanced Salt solution (Gibco) by removing the anterior segment, vitreous body, sclera and RPE and were frozen at $-80^{\circ} \mathrm{C}$. Retinas were lysed in P300 buffer $\left(20 \mathrm{mM} \mathrm{Na} \mathrm{NPO}_{4}\right.$; $250 \mathrm{mM} \mathrm{NaCl} ; 30 \mathrm{mM}$ NaPPi; 0.1\% Nonidet P-40; $5 \mathrm{mM}$ EDTA; $5 \mathrm{mM}$ DTT) supplemented with protease inhibitor cocktail (Sigma-Aldrich). For RPE protein extracts, the anterior segment and the retina were removed from enucleated mouse eyes. The RPE was then separated from the choroid by incubating the posterior eyecup (sclera-choroidRPE) with P300 buffer for $10 \mathrm{~min}$. Protein concentration 
was determined using a Lowry protein assay kit (DC Protein Assay; Bio-Rad). Equal amounts of proteins ( $20 \mu \mathrm{g} / \mathrm{lane})$ of each sample were loaded, separated by 7.5\% SDS-PAGE (Bio-Rad) and transferred onto nitrocellulose membranes. Western blots were then conducted using standard procedures. Primary and secondary antibodies are listed in Supplementary Table S2. An enhanced chemiluminescence kit (Bio-Rad) was used to detect the proteins. Each sample was probed once with anti- $\alpha$-tubulin antibody for normalisation. Quantification was done using Fiji software (National Institutes of $\mathrm{Health}^{24}$ ).

\section{Electroretinographic analysis}

Electroretinograms (ERGs) were recorded using a Micron IV focal ERG system (Phoenix Research Labs). Mice were dark-adapted overnight and prepared for recording in darkness under dim-red illumination. Mice were anesthetised with intraperitoneal injection of ketamine $(90 \mathrm{mg} / \mathrm{kg}$, Merial) and xylazine $(8 \mathrm{mg} / \mathrm{kg}$, Bayer $)$, and were topically administered tropicamide $(0.5 \%)$ and phenylephrine (2.5\%) for pupillary dilation. Flash ERG recordings were obtained from one eye. ERG responses were recorded using increasing light intensities ranging from -1.7 to $2.2 \log \mathrm{cd} . \mathrm{s} / \mathrm{m}^{2}$ under dark-adapted conditions, and from -0.5 to $2.8 \log \mathrm{cd} . \mathrm{s} / \mathrm{m}^{2}$ under a background light that saturates rod function. The interval between flashes varied from $0.7 \mathrm{~s}$ at the lowest stimulus strengths to $15 \mathrm{~s}$ at the highest ones. Five to thirty responses were averaged depending on flash intensity. Analysis of a-wave and b-wave amplitudes was performed using LabScribeERG software (https://www.iworx.com/ research/software/labscribe). The a-wave amplitude was measured from the baseline to the negative peak and the b-wave was measured from the baseline to the maximum positive peak.

\section{Fluorescein angiography}

Mice were anesthetised as described above. Pupils were dilated using $0.5 \%$ tropicamide (Théa) and 5\% chlorhydrate phenylephrine (Europhta) eye drops. The mouse was placed on the imaging platform of the Micron IV system (Phoenix Research Labs), and Ocry-gel was applied on both eye to keep the eye moist during the imaging procedure. Mice were injected in tail's vein with $100 \mu \mathrm{L}$ of $5 \%$ fluorescein isothiocyanate dextran (Sigma), and rapid acquisition of fluorescent images ensued for $\sim 5$ min.

\section{RNA extraction and RT-qPCR}

RT-qPCR experiment was performed on at least three mice per condition. Total RNA was extracted from a single retina using RNeasy mini kit (Qiagen) according to the manufacturer's instructions. RNA quantity was assessed using the NanoDrop 2000c UV-Vis spectrophotometer
(Thermo Fisher Scientific). Total RNA (500 ng) was reverse transcribed in the presence of oligo-(dT)20 using Superscript II reagents (Thermo Fisher Scientific). For each RT-qPCR, cDNA was used in the presence of EvaGreen (Bio-Rad), and the reactions were performed in triplicates on a CFX96 Real-Time PCR Detection System (Bio-Rad). Differential expression analysis was performed using the $\Delta \Delta \mathrm{Ct}$ method and normalised using the geometric mean expression of two housekeeping gene, Rps 26 and $\operatorname{Srp} 72^{25}$. For each gene, the relative expression in each sample was calculated using the mean of the controls as the reference (1 a.u.). Primers used are listed in Supplementary Table S1.

\section{Quantification and statistical analysis}

The numbers of labelled cells on retinal sections or retinal flat mounts were manually counted in a defined field (sizes are indicated in the legends). For all such counting, as well as qPCR and western blot analysis, at least three retinas were used per condition. The nonparametric one-tailed Mann-Whitney test was used for all statistical analysis implicating two unpaired groups. For the ERG experiment, where multiple statistical comparisons were needed, a two-way ANOVA followed by Bonferroni's multiple comparison test was used. All results are reported as mean \pm SEM. All analyses were performed using GraphPad Prism 8.3 (GraphPad Software, La Jolla California USA) with statistical significance set at $p$ value $\leq 0.05$.

\section{Results}

\section{Compensatory $\mathrm{Taz}$ regulation in $\mathrm{Yap}^{+/-}$postnatal mice}

In contrast with the developmental arrest observed in $\mathrm{Yap}^{-/-}$mice $^{18}$, Yap heterozygous mice are viable and fertile. We wondered whether Yap gene haploinsufficiency could occur in the adult, and therefore decided to examine, in detail, the molecular and phenotypic consequences of one Yap allele deletion. As expected, qPCR and western blot analysis confirmed an approximately two-fold decrease of Yap expression in postnatal and adult $\mathrm{Yap}^{+/-}$retinas compared to controls (Fig. 1a, b). By immuno-histochemical (IHC) analysis, we found that YAP expression was strongly diminished in both Müller glial and RPE cells, where YAP has previously been shown to be expressed $^{17}$ (Fig. 1c). Prior to analysing potential ocular phenotypes in $\mathrm{Yap}^{+/-}$mice, we wanted to confirm that the observed Yap expression reduction actually leads to the decrease of Yap target gene expression. Indeed, this may not be the case since compensatory regulation mechanisms have previously been reported between YAP and $\mathrm{TAZ}^{7,26,27}$. In accordance with this, we observed an increase in Taz transcript abundance in postnatal $\mathrm{Yap}^{+/-}$ retinas compared to controls (Fig. 1d). Noticeably, the compensatory upregulation waned in adult stages (after P21). This trend was confirmed at the protein level 

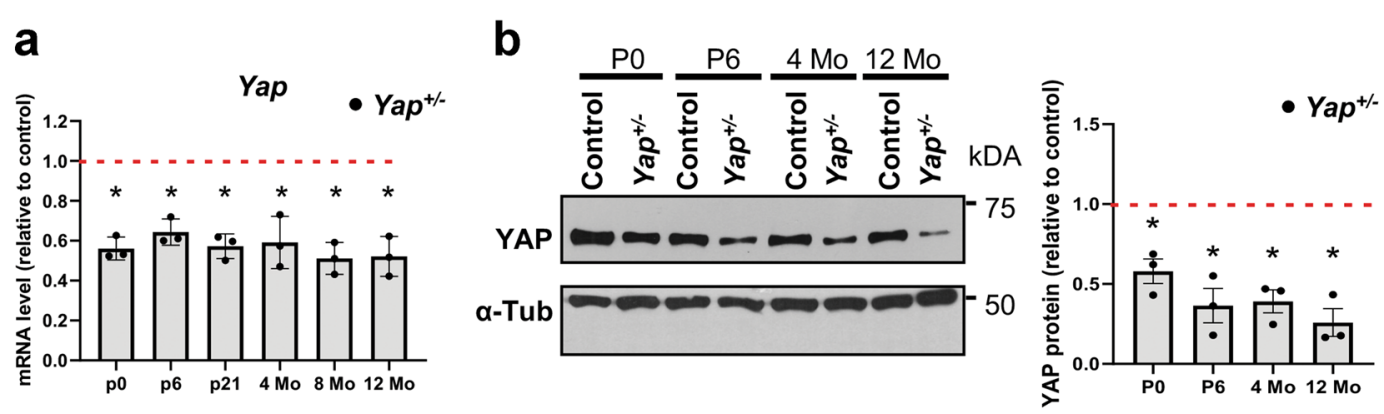

C
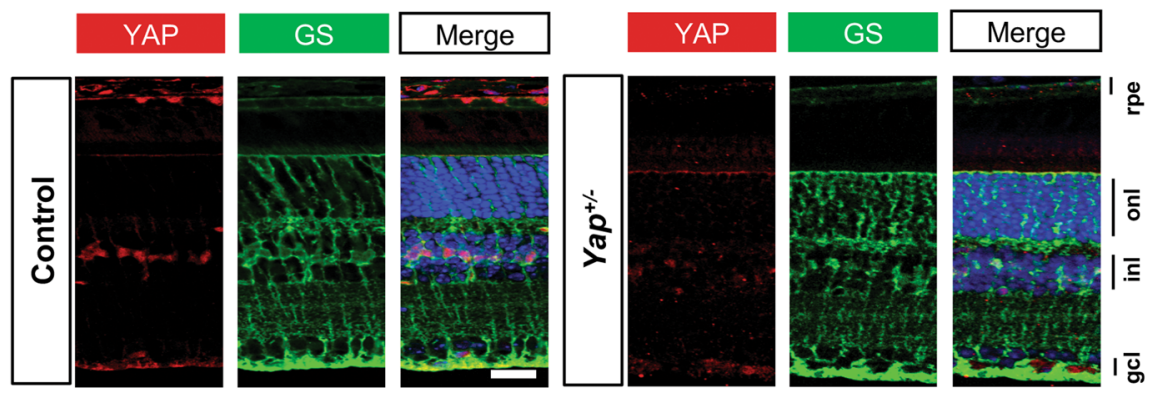

d
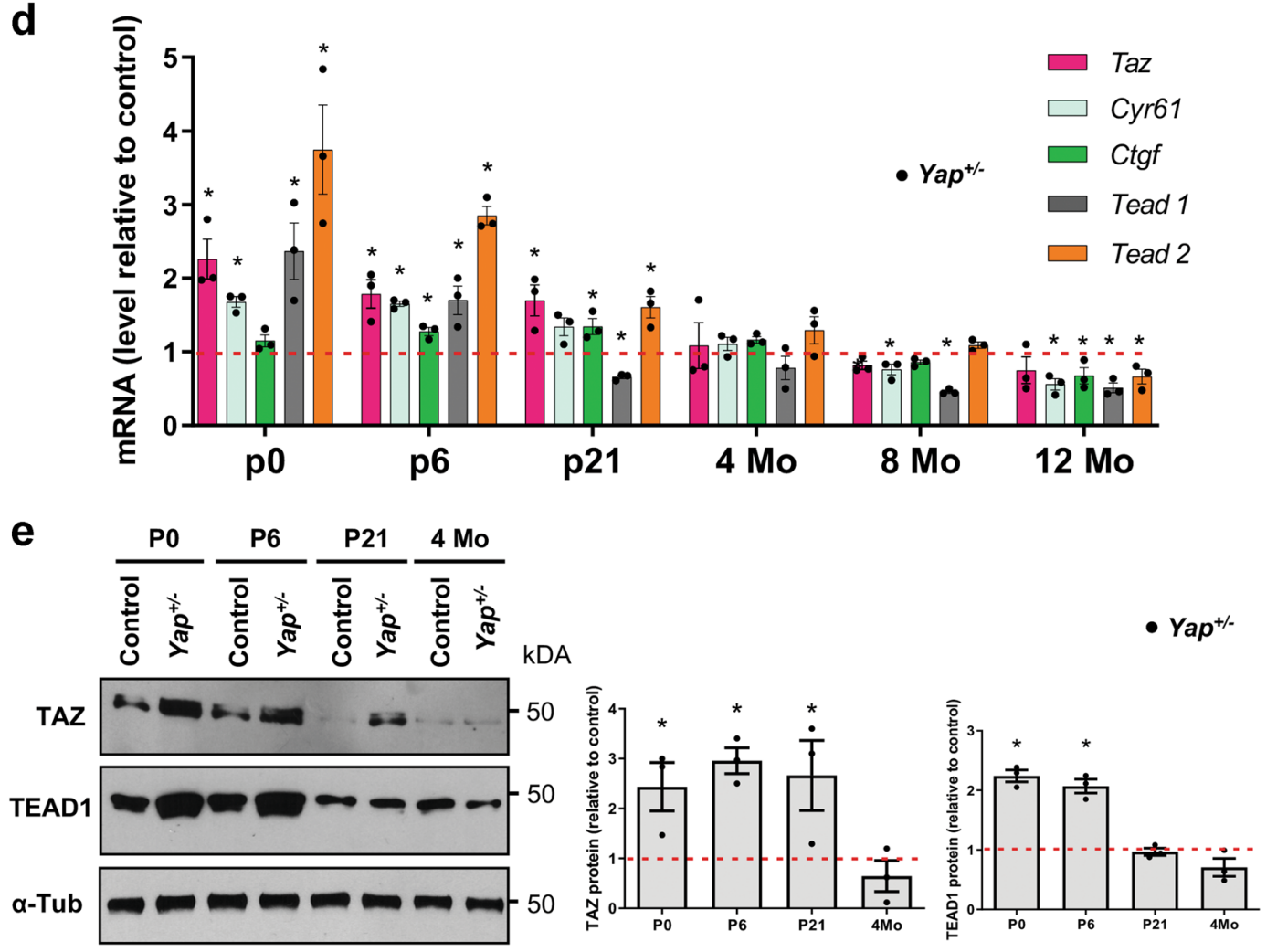

Fig. 1 Compensatory regulation in $\mathrm{Yap}^{+/-}$mice. a RT-qPCR analysis of Yap expression in Yap ${ }^{+/-}$mice retina, relative to wild-type controls (dashed line) ( $n=3$ biological replicates per condition). $\mathbf{b}$ Analysis of YAP protein expression level by western blot. The quantification is normalised to atubulin (a-Tub) signal and relative to wild-type controls at each stage (dashed line) ( $n=3$ biological replicates per condition). c 4-month-old mice retinal sections immunostained for YAP (red) and a marker of Müller cells, glutamine synthetase (GS, green). Nuclei are DAPI counterstained (blue). d RT-qPCR analysis of Taz, Cyr61, Ctgf, Tead1, and Tead2 expression relative to wild-type controls (dashed line) ( $n=3$ biological replicates per condition). e Western blots analysis of TAZ and TEAD1. The quantification is normalised to a-tubulin (a-Tub) signal and relative to controls at each stage (dashed line) ( $n=3$ biological replicates per condition). rpe: retinal pigment epithelium, inl: inner nuclear layer, onl: outer nuclear layer, gcl: ganglion cell layer. All values are expressed as the mean \pm SEM. Statistics: Mann-Whitney test, ${ }^{*} p \leq 0.05$. Scale bar: $20 \mu \mathrm{m}$. 
(Fig. 1e). In order to assess the net outcome of the compensatory mechanism on YAP/TAZ activity, we analysed by RT-qPCR the expression level of Ctgf and Cyr61, which have been recognised as direct YAP/TAZ target genes ${ }^{28}$. Similar to Taz expression profile, we found that both genes were first upregulated at postnatal stages (prior P21), but downregulated from 8-month onwards in $\mathrm{Yap}^{+/-}$mice compared to controls (Fig. 1d). YAP/TAZ interacting transcription factors TEAD1 and TEAD2 followed the same expression profile (Fig. 1d, e). Of note, TEAD3 and TEAD4 are not expressed in the mouse retina ${ }^{17}$. Together, these data reveal the existence of a dynamic regulatory mechanism taking place in $\mathrm{Yap}^{+/-}$mice, that first leads to an increased expression of key YAP related and target genes at postnatal stages due to the compensatory overexpression of TAZ that diminished progressively with aging, with a concomitant decreased expression of these genes in adult mice. This unexpected temporally dynamic compensation prompted us to undertake a thorough phenotypic analysis of $\mathrm{Yap}^{+/-}$mice retina at both postnatal and adult stages, when compensatory mechanisms are occurring or not, respectively.

\section{Delayed cell-cycle exit of retinal progenitor cells in $\mathrm{Yap}^{+/-}$ postnatal mice}

Yap overexpression in new-born mice was shown to promote retinal cell proliferation, while Yap knockdown leads to the opposite phenotype ${ }^{8}$. Therefore, considering the abundance changes of both YAP (decrease) and TAZ (increase) in Yap heterozygous postnatal retinas, we wondered how it impacts the proliferative behaviour of retinal progenitor cells. Interestingly, $24 \mathrm{~h}$ after EdU intraperitoneal injection at P5 (when most progenitors have exited the cell cycle), four times more EdU-positive cells were detected in the central part of mutant retinas compared to control ones (Fig. 2a, b). This persistence of a population of proliferative cells in $\mathrm{Yap}^{+/-} \mathrm{P} 6$ retinas was supported by IHC analysis, showing more cells labelled with the cell proliferation markers PCNA and Cyclin D1, compared to controls (Fig. 2c). We found no more EdUlabelled cells in $\mathrm{Yap}^{+/-}$retinas, neither in the central nor in the peripheral region, when EdU injection was performed at P11 (Supplementary Fig. S1), suggesting that $\mathrm{Yap}^{+/-}$late progenitors eventually exited the cell cycle between P6 and P11. We next sought to determine, by EdU birthdating, the fate of these late progenitor cells. Proliferative progenitors in P6 $\mathrm{Yap}^{+/-}$retinas gave rise to both late born neurons (CHX10-positive bipolar cells and Recoverin-positive photoreceptors) and glial cells (SOX9positive Müller cells) in the central retina (Supplementary Fig. S2A-D). To be able to compare this distribution with a control one, and since no EdU-positive cells were present in the central retina of P6 control individuals, we analysed the fate of EdU cells localised in the periphery of the retina. The proportion of double EdU/CHX10-, EdU/ Recoverin- and EdU/SOX9-positive cells among EdU cells was similar in $\mathrm{Yap}^{+/-}$and in control retinas (Supplementary Fig. S2E). Finally, a series of IHC staining on P21 animals did not reveal any significant differences between wild-type and $\mathrm{Yap}^{+/-}$retina regarding markers of rods, cones, bipolar, ganglion, amacrine, horizontal, and Müller cells (data not shown). Together, these data suggest that a subset of $\mathrm{Yap}^{+/-}$retinal progenitor cells have a delayed cell-cycle exit, without exhibiting any apparent bias in their cell fate determination.

\section{EGFR pathway potentiation in $\mathrm{Yap}^{+/-}$postnatal mice}

YAP has been recognised as an integrator of several key signalling pathways. In particular, crosstalk between EGFR and Hippo pathways have been reported ${ }^{22,29-31}$. Knowing that EGFR signalling regulates proliferation of retinal progenitor cells at postnatal stages ${ }^{32}$, we hypothesised that the excess of late retinal proliferative progenitor cells in $\mathrm{Yap}^{+/-}$retinas could result from a deregulated EGFR pathway. RT-qPCR analysis at P0 and P6 showed a significant upregulation of Egfr, Erbb2, $E r b b 3$, and Erbb4 (encoding receptors of the EGFR family: EGFR, Her2, Her3, and Her4, respectively), as well as of Hbegf and Neuregulin 1 (encoding EGFR ligands: HB-EGF and NRG1, respectively), in $\mathrm{Yap}^{+/-}$mice compared to controls (Fig. 3a). From P21, their expression diminished progressively in $\mathrm{Yap}^{+/-}$mice to reach control levels or levels lower than the controls (Fig. 3a). It is established that EGFR signalling acts through the activation of the MAPK, PI3K/AKT, or STAT3 pathways and previous reports have shown that these pathways are activated during proliferation of retinal progenitors cells ${ }^{33-35}$. We thus investigated whether their activity was affected in $\mathrm{Yap}^{+/-}$mice. P-AKT/AKT and P-STAT3/STAT3 ratios were increased in $\mathrm{Yap}^{+/-}$mice compared to controls at P6, while no difference was observed for P-ERK/ERK ratio (Fig. 3b). Together, these results revealed that EGFR pathway activity is potentiated at early postnatal stages in $\mathrm{Yap}^{+/-}$retina, and that this upregulation no longer occurs in adult stages. We therefore propose that the TAZ-dependent compensatory regulation that we observed in $\mathrm{Yap}^{+/-}$mice during postnatal stages may underlie this EGFR pathway activation.

\section{$\mathrm{Yap}^{+/-}$mice display retinal dysplasia}

We next sought to determine whether $\mathrm{Yap}^{+/-}$mice exhibit any ocular defect at adult stages when the TAZdependent compensatory regulation is no longer effective. Considering the delay in cell-cycle exit described above at postnatal stages, and because YAP/TAZ are implicated in the control of organ growth ${ }^{36,37}$, we examined the size of $\mathrm{Yap}^{+/-}$eyes. No major difference was found when compared to control eyes (Supplementary Fig. S3A). Cataract 


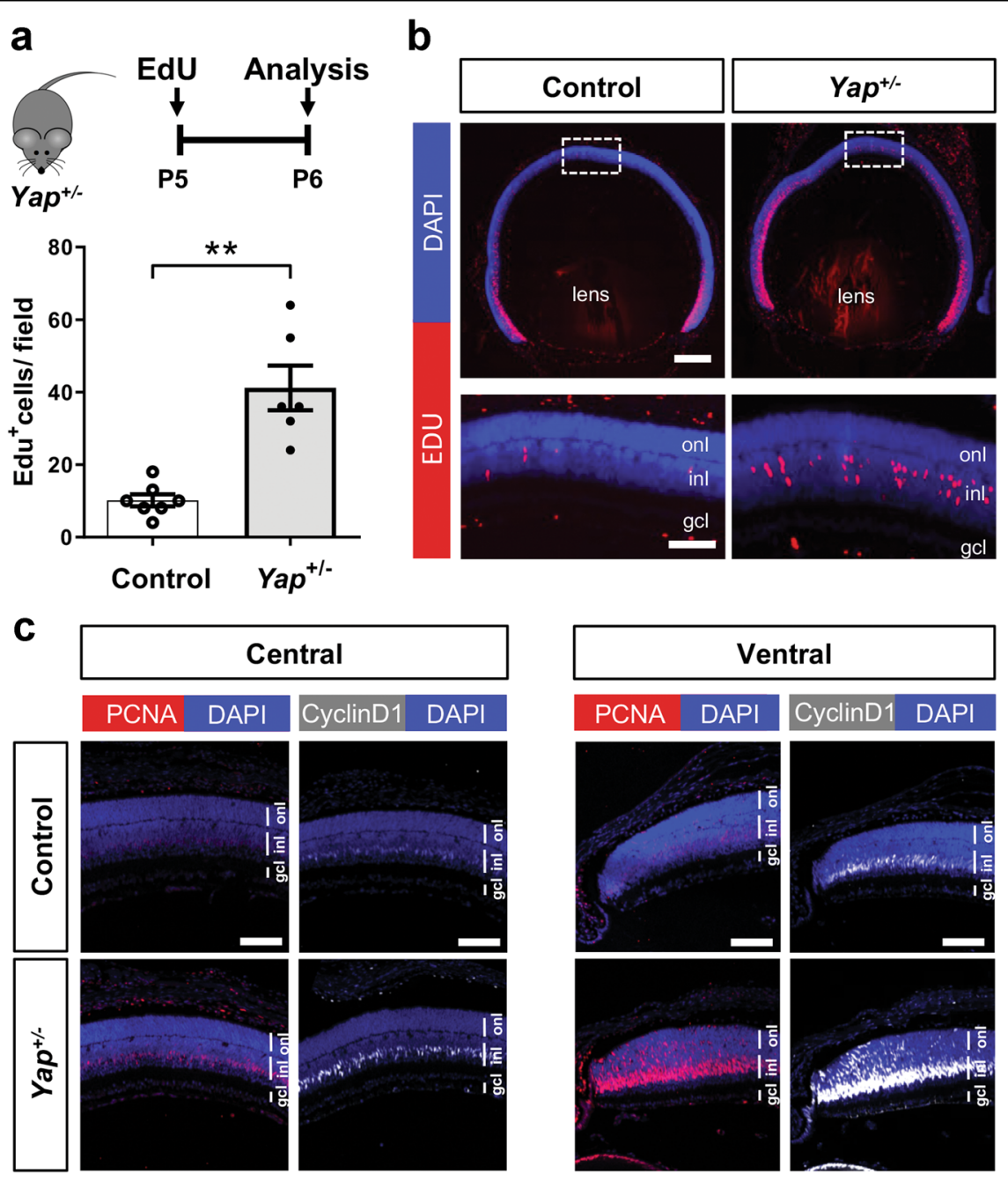

Fig. 2 Prolonged proliferation of retinal progenitors at postnatal stages in $\mathrm{Yap}^{+/-}$mice. a Timeline diagram of the experimental procedure used in b. Wild-type (Control) or Yap ${ }^{+/-}$mice were injected with EdU at P5 and analyzed $24 \mathrm{~h}$ later. b P6 retinal sections labelled for EdU (red) and stained with DAPI (blue). The delineated areas are enlarged in the bottom panels. Scatter plot with bars represents the number of EdU ${ }^{+}$cells per field $(250 \times 250 \mu \mathrm{m})$. Means \pm SEM from seven control retinas and six Yap ${ }^{+/-}$retinas are shown. c P6 retinal sections immunostained for PCNA (red) or Cyclin D1 (grey). Both central and peripheral ventral regions are shown. Nuclei are DAPI counterstained (blue). inl: inner nuclear layer, onl: outer nuclear layer, gcl: ganglion cell layer. Statistics: Mann-Whitney test, ${ }^{* *} p \leq 0.01$. Scale bar: $200 \mu \mathrm{m}$ (b) and $50 \mu \mathrm{m}$ (c and enlarged panels in b).

was occasionally observed but only in about $8 \%$ of $\mathrm{Yap}^{+/-}$ retinas (not shown). Heterozygous Yap loss-of-function in humans can result in coloboma ${ }^{15,16}$. We, however, did not detect any defects in optic fissure closure in $\mathrm{Yap}^{+/-}$ mouse retina (data not shown). Yet, from P21 onwards, we observed that some mutant retinas displayed one or two dysplastic regions in either the central or dorsal retina (Supplementary Fig. S3B-D). The incidence of this nonfully penetrant phenotype was higher in older mice (Supplementary Fig. S3D). The severity of the dysplasia was highly variable and each cell layer of the retina was susceptible to be affected (data not shown). Dysplasia were, however, never detected in the RPE and analysis of the expression of RPE cell markers suggests that the deletion of a Yap allele does not disturb RPE integrity (Supplementary Fig. S4A-C). Outside the dysplasia, no difference in the thickness of either the outer or the inner nuclear layers was observed between $\mathrm{Yap}^{+/-}$and control retinas (Supplementary Fig. S4D). This data indicates that the general structure of the adult retina is not affected in $\mathrm{Yap}^{+/-}$mice, apart from the dysplastic region.

\section{$\mathrm{Yap}^{+/-}$adult mice display progressive cone photoreceptor degeneration}

To assess the impact of one Yap allele deletion on adult retinal function, we performed electroretinogram (ERG) recordings in $\mathrm{Yap}^{+/-}$mice. Scotopic a- and b-waves were similar between controls and $\mathrm{Yap}^{+/-}$mice at all stages 


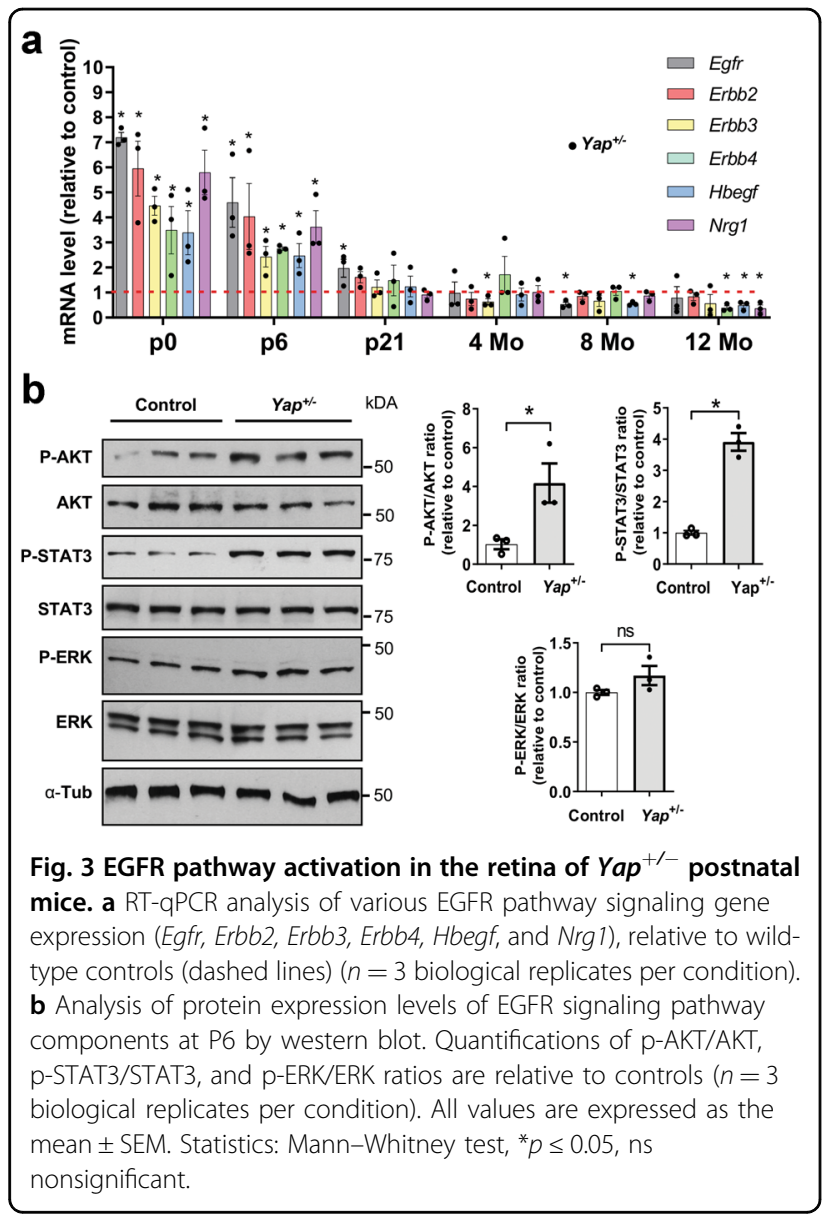

examined (Fig. 4a and Supplementary Fig. S5). This suggests that rod photoreceptors in $\mathrm{Yap}^{+/-}$mice function normally (reflected by the a-wave), and that visual signal is properly transmitted through the inner retina (reflected by the b-wave). Strikingly however, photopic b-wave amplitude presented a significant depression in 12-month-old $\mathrm{Yap}^{+/-}$mice compared to controls at high intensity stimuli (Fig. 4a). Such specific reduction of the cone-mediated ERG response could signify either defects in the synaptic transmission between cone photoreceptors and bipolar cells and/or the presence of a cone dystrophy. In 12month-old $\mathrm{Yap}^{+/-}$retina, IHC analysis showed a significant decrease in the number of Ribeye-positive puncta, which labels the presynaptic ribbons in photoreceptor terminals (Supplementary Fig. S6). Ribbons with proper horseshoe shape were present close to dendritic process of the rod-bipolar cell postsynaptic terminals, labelled with anti-Protein Kinase $C$ alpha (PKC- $\alpha$ ), suggesting a correct synaptic connection between rod photoreceptor and rodbipolar cells in $\mathrm{Yap}^{+/-}$retina. In contrast, some ribbons did not exhibit the typical horseshoe shape suggesting compromised synapse integrity (Supplementary Fig. S6). We next assessed whether these defects were associated with cone photoreceptor defects in $\mathrm{Yap}^{+/-}$retina. The number of cones, as inferred by peanut agglutinin (PNA) labelling, was significantly decreased in $\mathrm{Yap}^{+/-}$ventral retinas compared to controls, affecting both S-opsin and M-opsin labelled cones (Fig. 4b, c). S- and M-opsin labelling were indeed both reduced in the ventral retina. Sopsin labelling was also severely decreased in the middorsal (the most dorsal part was not analysed considering the occurrence of dysplasia) and the central retina. In addition to this quantitative phenotype, we also found that the remaining staining was abnormal, being more punctuated compared to the fusiform fluorescence in controls. This phenotype is reminiscent of degenerative cones ${ }^{38}$. We confirmed on flat-mounted retinas the punctuated versus the fusiform labelling, as well as the reduced number of PNA, S-opsin, and M-opsin labelled cones in the ventral region of 12-month-old $\mathrm{Yap}^{+/-}$mutant mice (Supplementary Fig. S7A). Consistent with a cone degenerative phenotype, we found that Cone Arrestin labelling was also severely decreased in the ventral retina (Supplementary Fig. S7B). The cone phenotype was only detected in old mice, as no difference in PNA, S- or M-opsin expression was observed between control and 1- or 4-month-old $\mathrm{Yap}^{+/-}$mice (Supplementary Fig. S8), consistent with the ERG data. Regarding rods, Rhodopsin was localised as expected within rod outer segments and we did not detect any major difference in its distribution between 12-monthold mutant and control retinas (Supplementary Fig. S9A). This result also supports the ERG data showing no rod dysfunction. Altogether, these results demonstrate that heterozygous mutation of Yap leads to specific cone dystrophy in aged mice.

\section{Deregulation of genes important for Müller cells homeostasis in $\mathrm{Yap}^{+/-}$mice}

Since YAP is expressed in adult Müller cells ${ }^{17}$ and Müller cells maintain retinal homeostasis ${ }^{39}$, we wondered whether cone degeneration in $\mathrm{Yap}^{+/-}$mice could result from altered Müller cell function. First, we showed that Müller cells were properly located within the inner nuclear layer and that their number was not changed in mutant mice (Supplementary Fig. S9B). However, RTqPCR, western blot and IHC analyses revealed that GFAP (glial fibrillary acidic protein) expression, the most sensitive indicator of retinal stress in Müller cells, was dramatically increased in 12-month-old $\mathrm{Yap}^{+/-}$retina compared to controls (Fig. 5a-c). Therefore, we next explored the impact of Yap heterozygous mutation on the expression of Müller cell-specific homeostatic regulatory proteins, aquaporin-4 (AQP4) and the potassium channel Kir4.1. Although no significant difference was observed at 8 months, we found that Kir4.1 and AQP4 protein levels were severely reduced in $\mathrm{Yap}^{+/-}$mice compared to controls at 12 months of age (Fig. 5d, e). Since Müller cells 

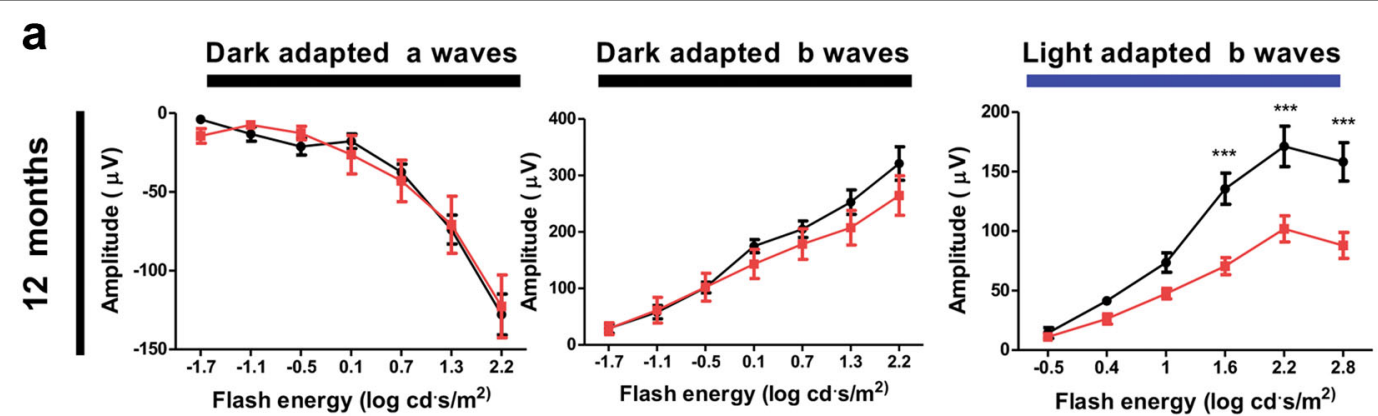

b
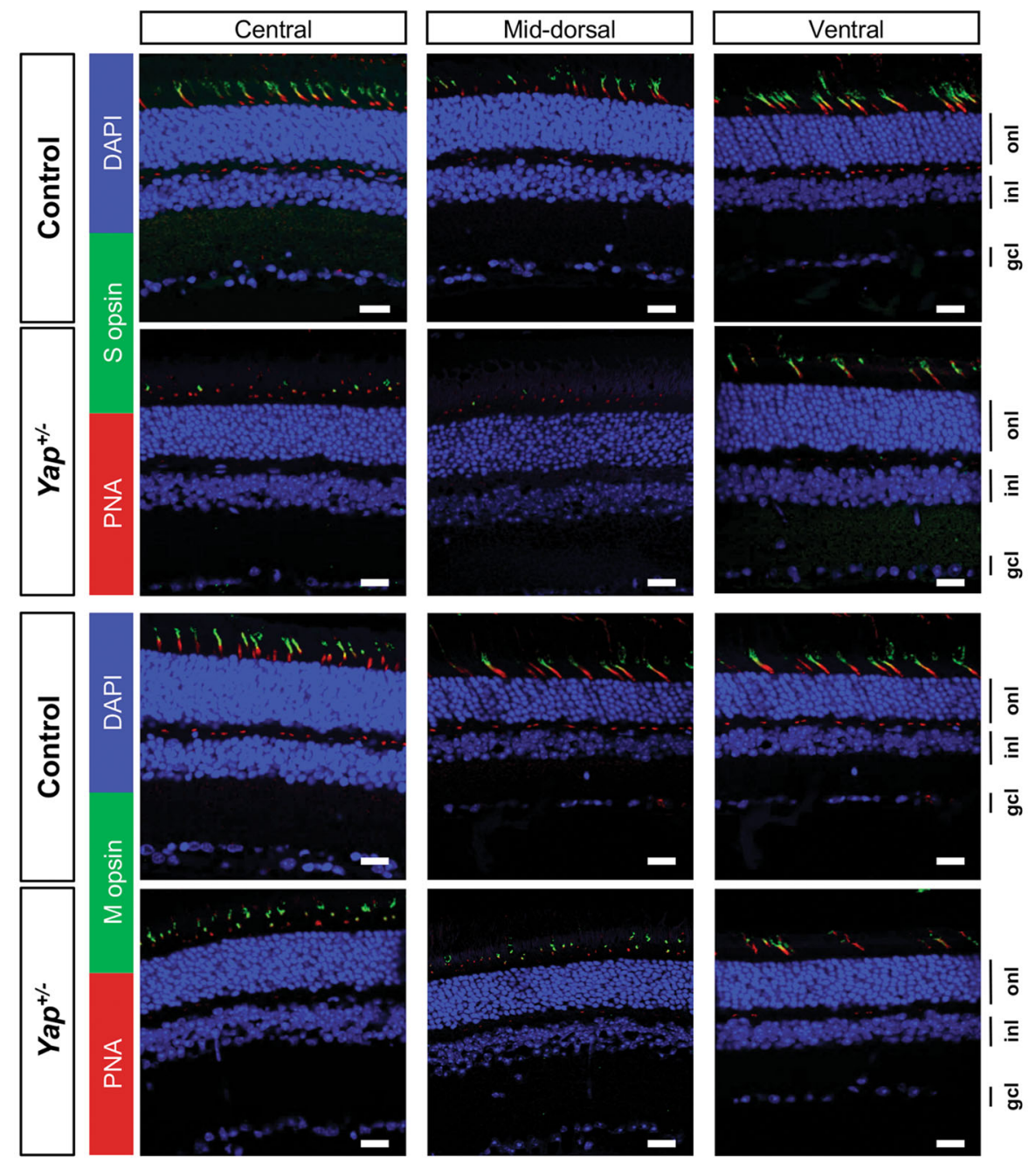

C
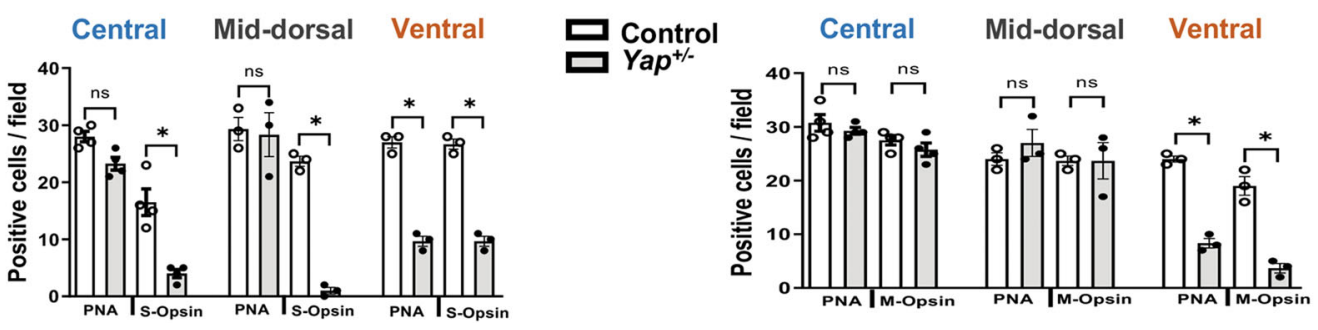

Fig. 4 (See legend on next page.) 
(see figure on previous page)

Fig. 4 Altered cone-driven vision and decreased expression of cone photoreceptor markers in $\mathrm{Yap}^{+/-}$adult mice. a Quantitative evaluation of the scotopic and photopic a- and b-waves maximum amplitude data from 12-month-old wild-type (black) or Yap ${ }^{+/-}$(red) mice. Mean \pm SEM intensity response curves are averaged from nine controls and seven $Y_{a p^{+/-}}$mice. Statistics: two-way ANOVA test, ${ }^{* * *} p \leq 0.001$. b Retinal sections from 12-month-old wild-type (Control) and $\mathrm{Yap}^{+/-}$mice, immunostained for cone makers (PNA, S-Opsin, and M-Opsin). Central, mid-dorsal, and ventral regions of retinal sections are shown. Nuclei are DAPI counterstained (blue). c Scatter plots with bars represent the number of labelled cells per field $(300 \times 300 \mu \mathrm{m})$. Values are expressed as the mean \pm SEM from 3 to 4 retinas per condition. onl: outer nuclear layer, inl: inner nuclear layer, gcl: ganglion cell layer. Statistics: Mann-Whitney test, ${ }^{*} p \leq 0.05,{ }^{* *} p \leq 0.01$, ns nonsignificant. Scale bars: $20 \mu \mathrm{m}$.

have been shown to actively participate at cone opsins recycling ${ }^{40}$, we wondered whether the expression of conespecific visual cycle factors could be altered in $\mathrm{Yap}^{+/-}$ Müller cells. We thus analysed the expression of cellular retinaldehyde-binding protein (CRALBP), given its key role in cone visual cycle and its expression in Müller cells ${ }^{41,42}$. We observed a reduction of CRALPB labelling in both Müller glia and RPE cells of 12-month-old $\mathrm{Yap}^{+/-}$ retinas, compared to controls (Fig. 6).

Perturbed ionic channels, such as the potassium channel Kir4.1, in Müller cells is a leading cause of intraretinal blood vessel defects ${ }^{43}$. We thus examined the three retinal vascular plexi in 12-month-old $\mathrm{Yap}^{+-}$mice (Supplementary Fig. S10A). We observed a reduction of the intermediate vascular plexus staining, without apparent blood leakage in 12-month-old $\mathrm{Yap}^{+/-}$retinal flatmounts, compared to controls (Supplementary Fig. S10B and Supplementary Fig. S11). Although recent studies have shown that YAP/TAZ are involved in vascular retinal development ${ }^{44}$, no obvious differences could be observed after labelling the three capillary plexi between 8-month-old $\mathrm{Yap}^{+/-}$mice and controls (Supplementary Fig. S10B). Such observation ruled out significant developmental defects of vascular networks. Collectively, these data revealed some retinal vasculature defects and altered Müller cell homeostatic function in $\mathrm{Yap}^{+/-}$aged mice.

\section{Conditional Yap deletion in Müller glia leads to Müller cell homeostasis dysfunction and altered pattern of cone opsin expression}

We next sought to investigate whether Müller glia could be the main cell type in which Yap haploinsufficiency mediates the observed cone degenerative phenotype. We took advantage of a transgenic line that we previously generated, Yap ${ }^{f l o x} / f l o x ; R a x-C r e E R^{T 2}$, and that allows Cremediated conditional gene ablation specifically in Müller cells $^{22}$. It is thereafter named Yap CKO while "control" refers to Yap ${ }^{\text {floxfllox }}$ mice. Yap deletion was induced in fully differentiated Müller cells, through 4-OHT intraperitoneal injection at P10 (Fig. 7a). Phenotypic analyses were then conducted on 12-month-old mice. Similar to what we observed in $\mathrm{Yap}^{+/-}$aged mice, the expression of Müller cell-specific homeostatic regulatory proteins AQP4 and Kir4.1 were downregulated and GFAP upregulation indicated reactive gliosis (Fig. 7b). Moreover, the expression pattern of S- and M-cone opsins was severely affected compared to control retinas, S-opsin signal being drastically decreased dorsally while $\mathrm{M}$-opsin signal being almost absent ventrally in Yap CKO mice (Fig. 7c, d). This phenotype is similar to that observed in $\mathrm{Yap}^{+/-}$aged retinas. However, the number of cones, as inferred by PNA labelling, was not significantly changed in Yap CKO mice compared to controls (Fig. 7c, d). Together, although the phenotype appears less severe in Yap CKO retinas than in $\mathrm{Yap}^{+/-}$mice, the sole deletion of Yap in Müller cell triggers defects in cone opsin expression. These data thus led us to propose a model in which YAP function in Müller cells would be necessary for ensuring the maintenance of Müller cell homeostasis and preserving cone integrity (Fig. 8).

\section{Discussion}

While YAP function during eye development has been well documented, our data demonstrate the usefulness of Yap heterozygous mice to better understand YAP function in the adult retina. Interestingly, our study revealed that the compensatory upregulation of YAP partner, TAZ, could not be sustained throughout life and declined during aging. This dynamic regulatory mechanism results in Yap haploinsufficiency only in the aged retina. We propose a model where the maintenance of proliferative progenitor cells in $\mathrm{Yap}^{+/-}$postnatal retina would result from the compensatory TAZ activity, leading to a gain of function-like phenotype, mediated by EGFR pathway potentiation (Fig. 8). During aging, when this compensatory mechanism is no longer operational in $\mathrm{Yap}^{+/-}$mice, we discovered the occurrence of an age-related cone dystrophy, associated with reduced cone-driven vision. Our data suggest that this phenotype may be the consequence of impaired Müller cell homeostatic function. This study therefore reveals that deregulation of Yap gene dosage could cause retinal degenerative diseases.

It has previously been reported that changes in YAP abundance result in compensatory regulation of TAZ to maintain Hippo signalling homeostasis ${ }^{45}$. We interestingly found that one Yap allele deletion was indeed accompanied by an increase in Taz expression in the retina, as well as an increase in Tead gene expression. However, 

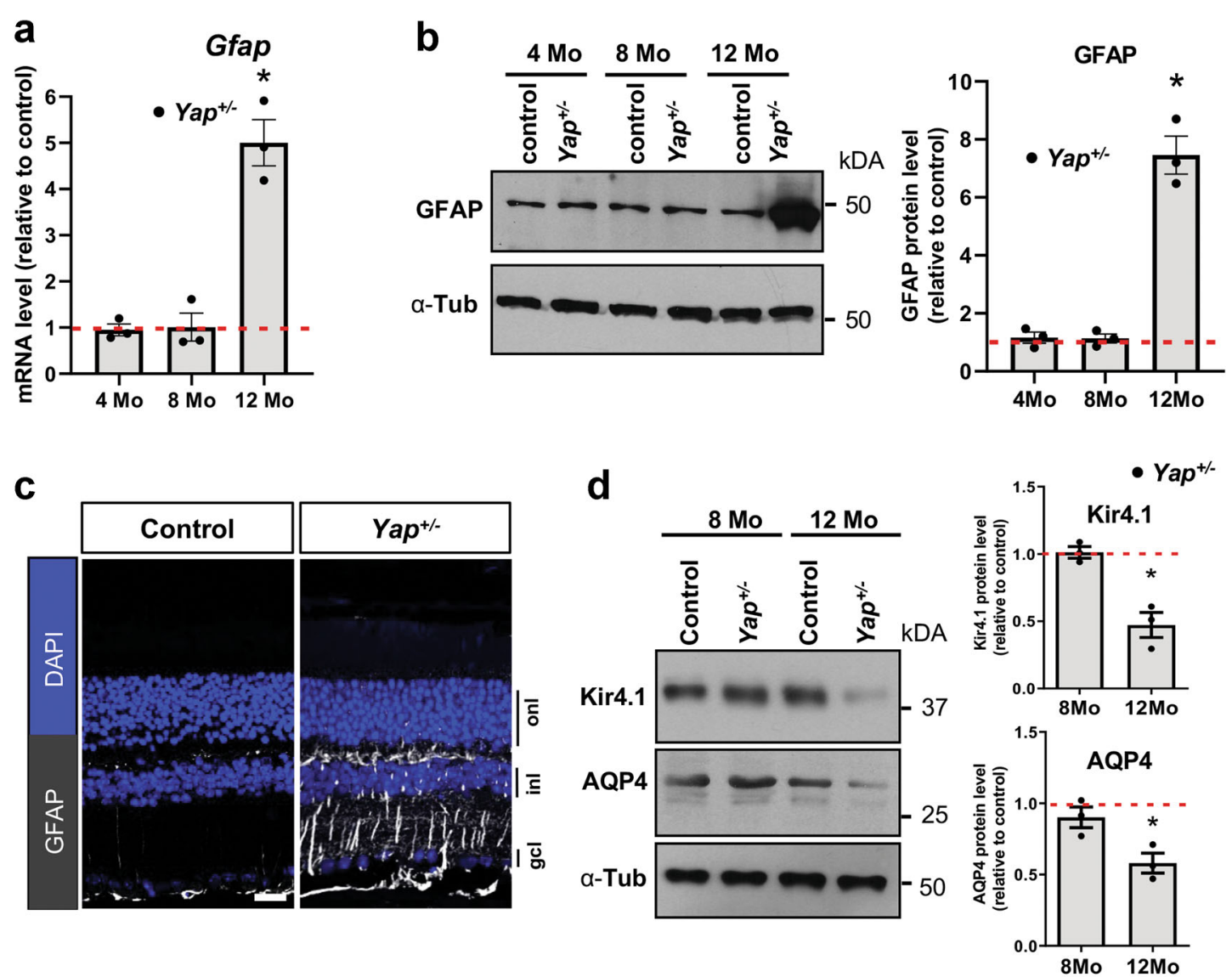

e

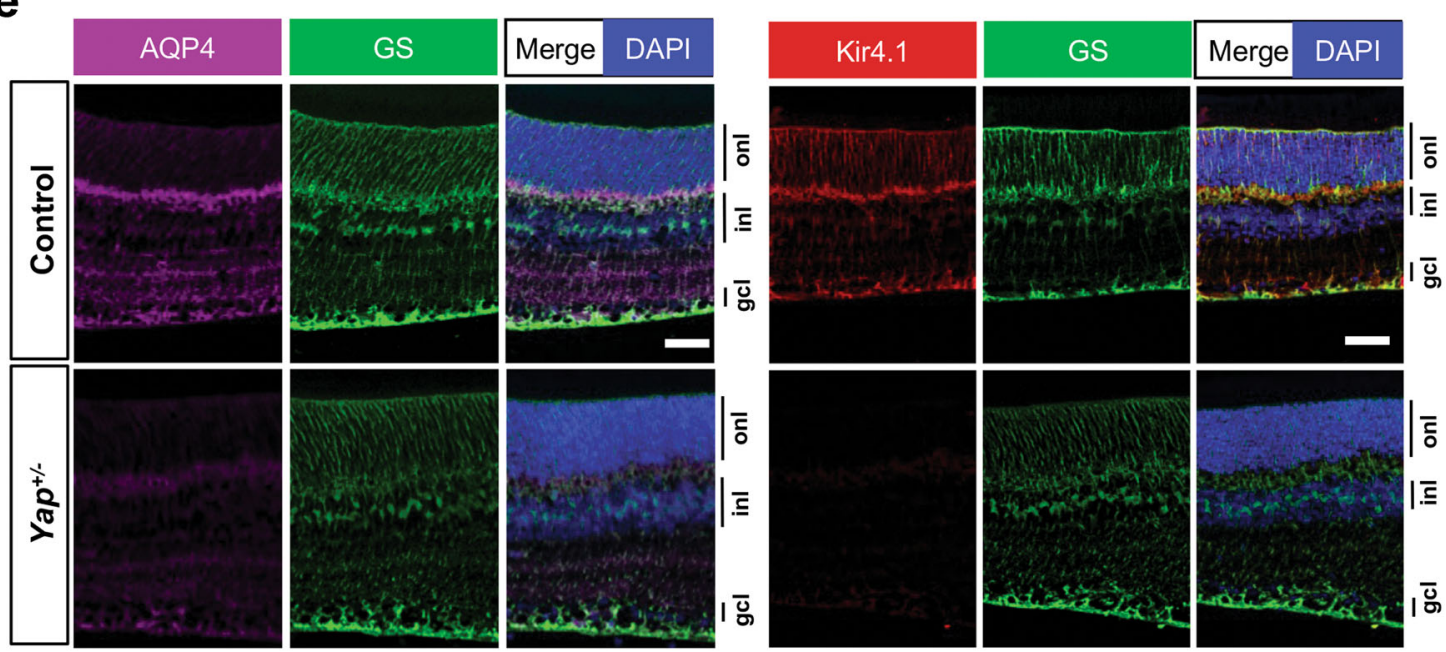

Fig. 5 Altered Müller glia homeostasis in Yap ${ }^{+/-}$adult mice. a RT-qPCR analysis of Gfap expression, relative to wild-type controls (dashed line) ( $n=3$ biological replicates per condition). b Analysis of GFAP protein expression level by western blot. Results are normalised to a-tubulin (a-Tub) signal and expressed relative to controls at each stage (dashed line) ( $n=3$ biological replicates per condition). c 12 -month-old retinal sections immunostained for GFAP (white). Nuclei are DAPI counterstained. $\mathbf{d}$ Analysis of Kir4.1 and AQP4 protein expression levels by western blot, in 8 or 12-month-old mice. Results are normalised to a-tubulin (a-Tub) signal and expressed relative to controls at each stage (dashed line) ( $n=3$ biological replicates per condition). e 12-month-old retinal sections immunostained for Kir4.1 (red), AQP4 (purple), or the Müller cell marker glutamine synthetase (GS, green). Nuclei are DAPI counterstained (blue). onl: outer nuclear layer, inl: inner nuclear layer, gcl: ganglion cell layer. All values are expressed as the mean \pm SEM. Statistics: Mann-Whitney test, ${ }^{*} p \leq 0.05$. Scale bar: $50 \mu \mathrm{m}$. 

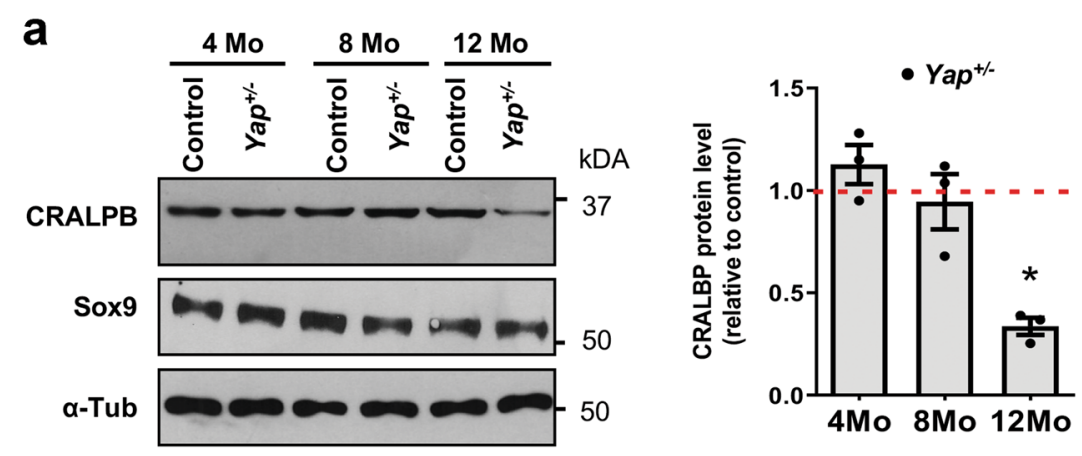

b
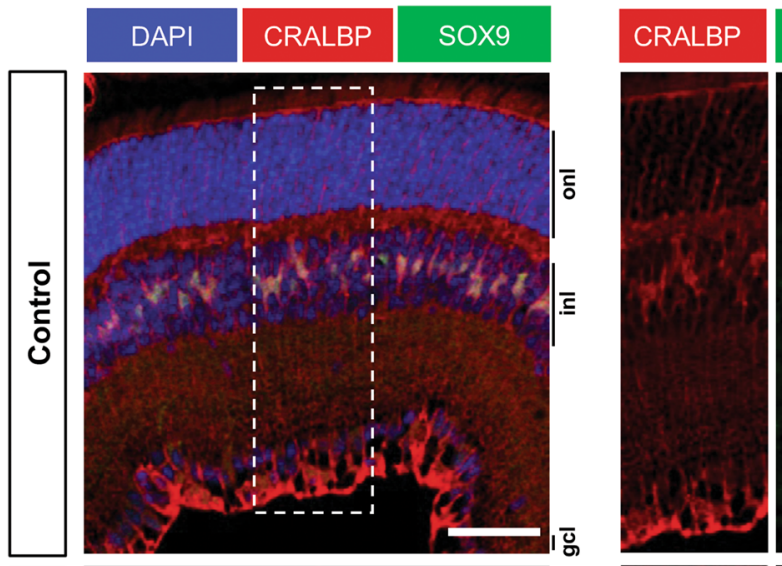

\section{sox9}
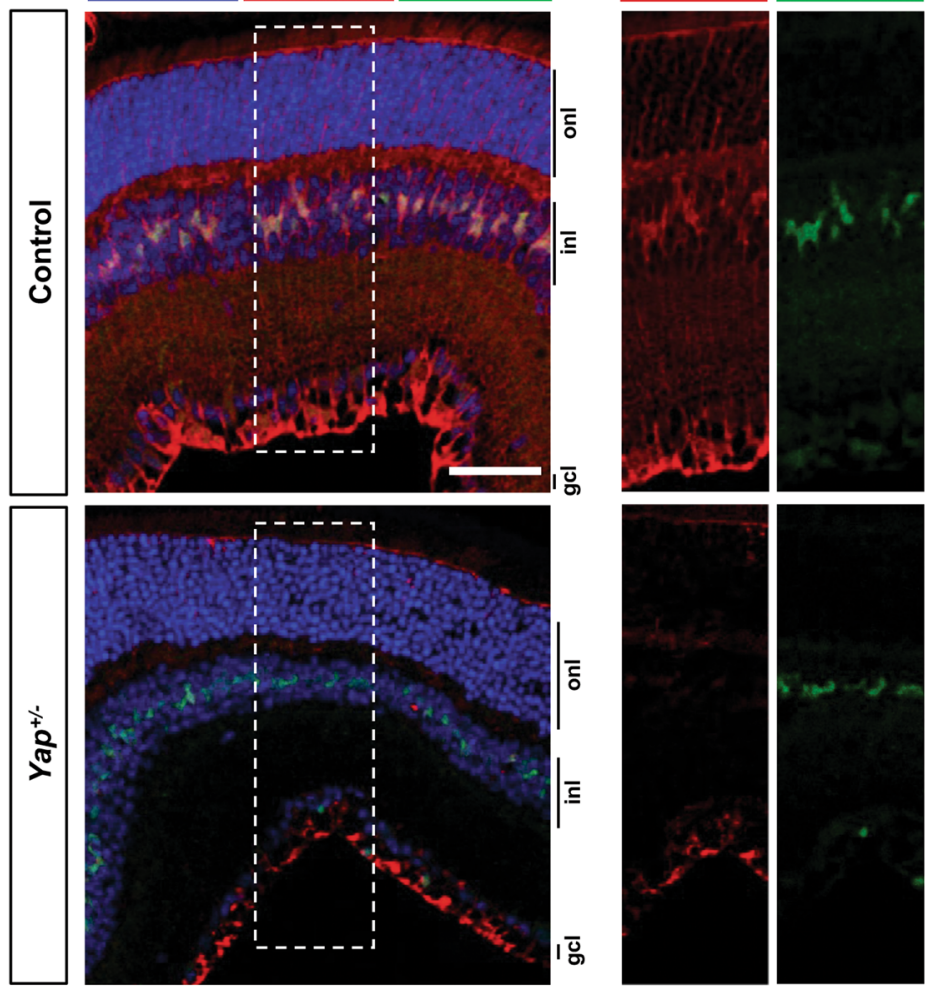

$\mathbf{C}$
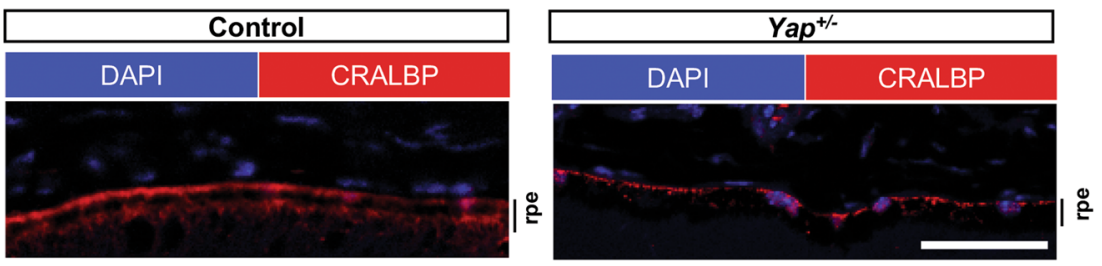

Fig. 6 Decreased CRALBP expression in $\mathrm{Yap}^{+/-}$adult mice retina. a Analysis of CRALBP protein expression level by western blot. SOX9 serves as a marker of Müller cells. Results are normalised to a-tubulin ( $a$-Tub) signal and expressed relative to controls at each stage (dashed line) ( $n=3$ biological replicates per condition). Values are expressed as the mean \pm SEM. b 12-month-old retinal sections immunostained for CRALBP (red) and SOX9 (green). Nuclei are DAPI counterstained (blue). The delineated areas (dashed lines) are enlarged in the right panels. c 12-month-old RPE sections immunostained for CRALBP (red). Nuclei are DAPI counterstained (blue). inl: inner nuclear layer, onl: outer nuclear layer, gcl: ganglion cell layer, rpe: retinal pigment epithelium. Statistics: Mann-Whitney test, ${ }^{*} p \leq 0.05$. Scale bar: $50 \mu \mathrm{m}$ (b) and $100 \mu \mathrm{m}$ (c).

such rises appeared to go beyond Yap compensation as we observed an increase in YAP/TAZ target genes. Interestingly, this dosage compensation system was not maintained in the retina of adult mice after 4 months of age. All these quantitative data on the abundance of YAP/ TAZ and their target genes highlight $\mathrm{Yap}^{+/-}$mice as an 

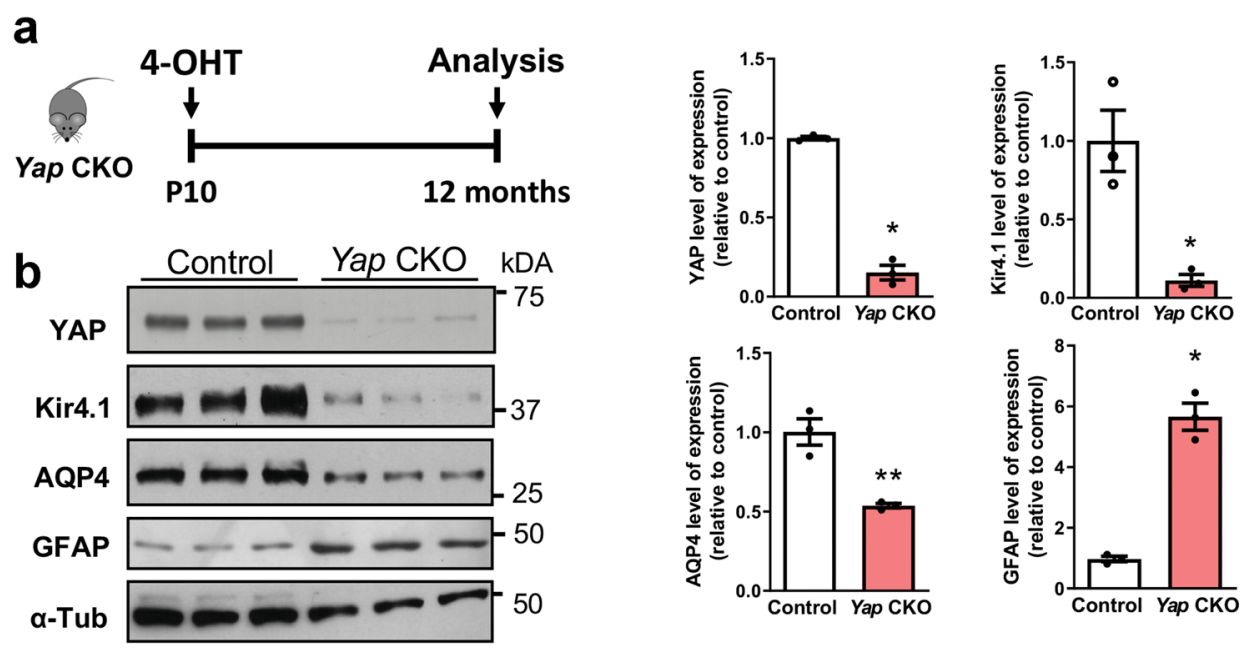

C

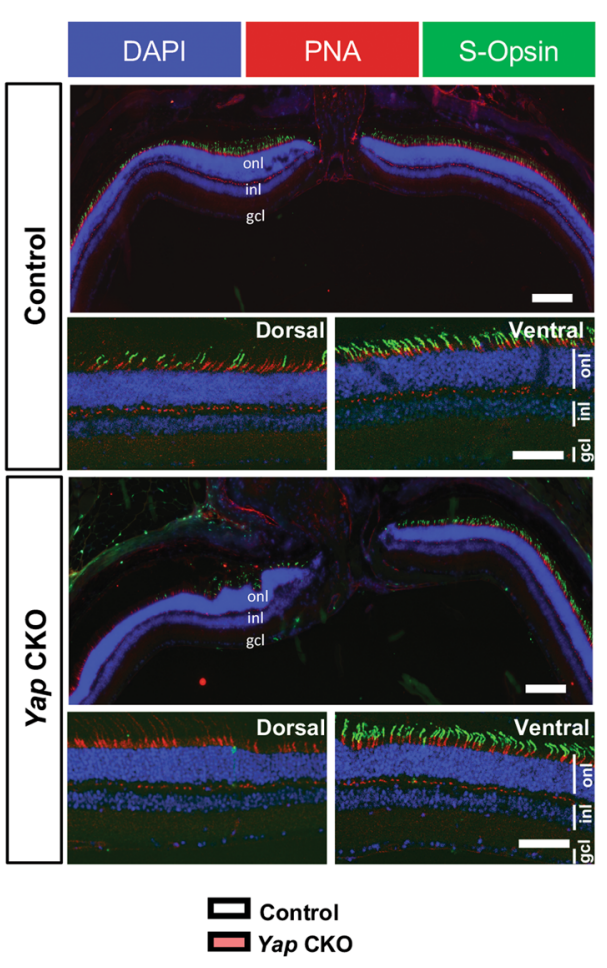

d
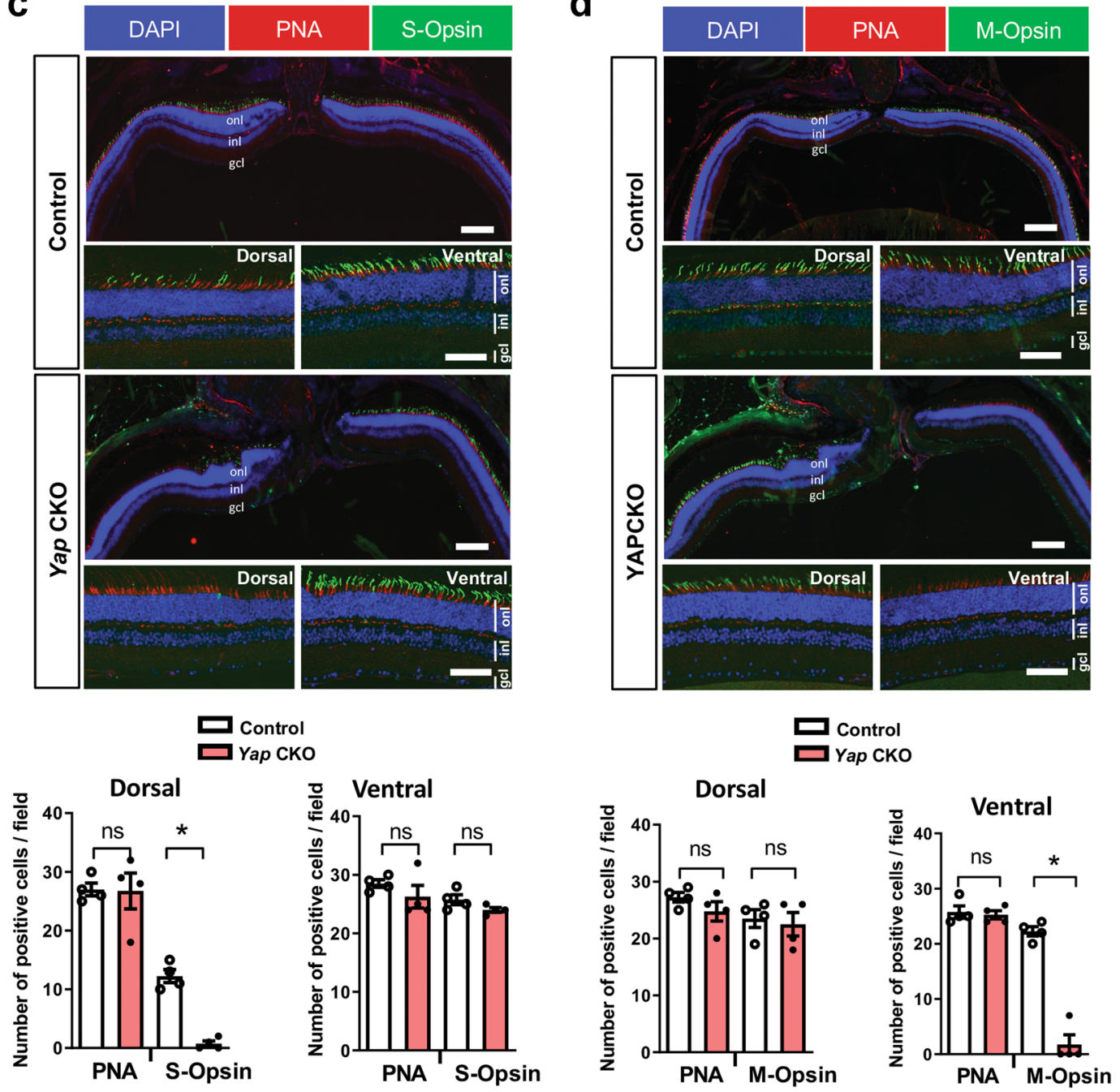

Fig. 7 (See legend on next page.) 
(see figure on previous page)

Fig. 7 Müller cell dysfunction and decreased expression of S/M-opsin in Yap CKO. a Timeline diagram of the experimental procedure used in b. Yap floxflox mice (control) and Yap floxflox; Rax-CreER ${ }^{T 2}$ mice (Yap CKO) received a single dose of 4-OHT at P10 and their retinas were analyzed at 12 months. b Analysis of the protein expression level of AQP4, Kir4.1, and GFAP. Results are normalised to a-tubulin (a-Tub) signal and expressed relative to control ( $n=3$ biological replicates per condition). $\mathbf{c}$, $\mathbf{d}$ Retinal sections from 12-month-old control and Yap CKO mice immunostained for S-opsin (green) and PNA (red) (c) or M-opsin (green) and PNA (red) (d). Dorsal, central and ventral sections of retinal sections are shown. Nuclei are DAPI counterstained (blue). Scatter plots with bars represent the mean number of labelled cells per field $(250 \times 250 \mu \mathrm{m})$ from four retinas per condition. All values are expressed as the mean \pm SEM. onl: outer nuclear layer, inl: inner nuclear layer, gcl: ganglion cell layer. Statistics: Mann-Whitney test, ${ }^{*} p \leq 0.05,{ }^{* *} p \leq 0.01$, ns nonsignificant. Scale bars: $100 \mu \mathrm{m}$ (central part) (enlarged panels) and $50 \mu \mathrm{m}$ (dorsal and ventral part).
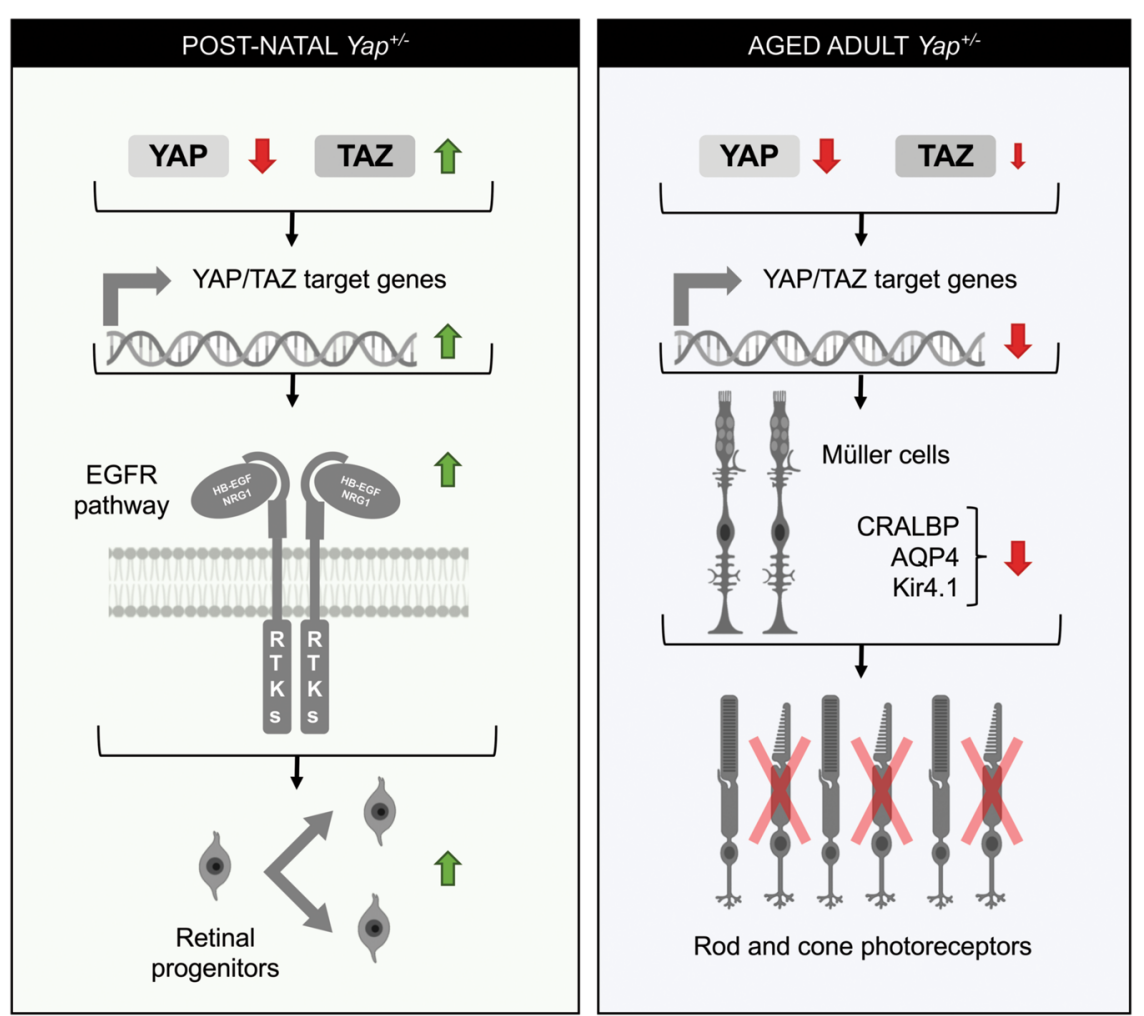

Fig. 8 Proposed model explaining $\mathrm{Yap}^{+/-}$phenotypes at postnatal and adult stages. Our results revealed the occurrence of a TAZ compensatory mechanism in postnatal Yap ${ }^{+/-}$retina, i.e., the decreased of Yap expression (red arrow) is accompanied by an increase of Taz expression (green arrow), that results in the upregulation of YAP/TAZ target gene expression. Our data suggest that this leads to prolonged proliferation of postnatal retinal progenitor cells, and that this phenotype may likely result from EGFR pathway potentiation. In the adult Yap ${ }^{+/-}$ retina, the compensatory mechanism is no longer effective, and YAP/TAZ target genes expression is diminished. Under such conditions, one Yap allele deletion progressively leads to impaired Müller cell homoeostasis. We propose that this may, at least in part, contribute to impaired conespecific visual cycle and therefore to the cone dystrophy observed in aged $\mathrm{Yap}^{+/-}$mice. RTKs Receptor tyrosine kinases. This figure was created with some schemas from @BioRender-biorender.com.

excellent model to study the role of YAP in the adult retina, when TAZ no longer compensates for Yap deletion. Unlike in humans, where heterozygous loss-of-function mutations in YAP causes coloboma ${ }^{15,16}, \mathrm{Yap}^{+/-}$mice do not exhibit such congenital malformation of the eye. A plausible hypothesis is that the compensatory mechanism during eye development is not regulated in humans as it is in mice. Interestingly, the phenotype in humans is not fully penetrant, which could indicate potential YAP/TAZ dosage variations between individuals.
YAP overexpression in neonatal mouse retinas was shown to promote cell proliferation and inhibit cell-cycle exit of late retinal progenitors ${ }^{8}$. We also found a delay in cell-cycle exit in $\mathrm{Yap}^{+/-}$postnatal retina. We propose that this is the consequence of the compensatory regulation that leads to TAZ level increase. Indeed, despite the genetic $\mathrm{Yap}^{+-}$context, we observed an upregulation of YAP/TAZ target genes at postnatal stages. Since the observed delayed cell-cycle exit does not appear to cause an overall increase in cell number, it may result from 
slower cell-cycle kinetics. We have previously shown in Xenopus retina that Yap knockdown results in perturbed cell-cycle kinetics of retinal stem cells in the ciliary mar$\operatorname{gin}^{46}$. Further analyses are, however, required to measure cell-cycle length in $\mathrm{Yap}^{+/-}$retinal progenitors. Furthermore, our data suggest that this delayed cell-cycle exit of retinal progenitor may be mediated by an increase in EGFR activity. Previous studies in other cell types have demonstrated that YAP and TAZ promote proliferation by potentiating the EGFR pathway ${ }^{29-31}$. This is also consistent with our recent finding suggesting that YAP is a key regulator of the EGFR pathway in adult reactive Müller cells in a degenerative context and that EGFR pathway is required for YAP mitogenic activity ${ }^{22}$. It remains to be investigated whether EGFR pathway components are directly regulated by YAP/TAZ in retinal cells. We raised the hypothesis that the prolonged maintenance of cell proliferation at postnatal stages could be linked to the appearance of dysplastic regions in $\mathrm{Yap}^{+/-}$retina. Indeed, the maintenance of progenitor cells in proliferation may subsequently lead to abnormal folding of retinal layers. Alternatively, considering that YAP regulates key effectors of matrix stiffening ${ }^{47}$, we can also hypothesise that the stiffness of the retinal tissue is affected, leading to retinal misfolding. This could underlie the increased incidence of dysplasia observed during aging.

We showed previously that YAP is expressed only in Müller cells in the adult retina ${ }^{17}$. We thus raised the hypothesis that cone degeneration in $\mathrm{Yap}^{+/-}$mice could result from altered Müller cell function. Indeed, Müller cells play many essential roles in retinal maintenance, regulation of synaptic activity, ion, and water homeostasis, and, importantly, in mediating cone visual cycle ${ }^{39}$. Moreover, conditional Müller cell ablation was shown to cause photoreceptor degeneration ${ }^{48,49}$. Our hypothesis is supported by the finding that deleting Yap specifically in Müller cells in Yap CKO mice also leads to abnormal expression of cone opsins. Both mutants exhibit reactive gliosis and impaired expression of genes involved in Müller cell homeostatic function (AQP4 and Kir4.1) consistent with a model where impaired cone integrity would be caused by Müller cell dysfunction.

We also discovered in $\mathrm{Yap}^{+/-}$Müller glia an altered expression of CRALBP, which is involved in cone-specific visual cycle. Knockdown or mutations in CRALBP gene lead to decreased cone-driven ERG responses in zebrafish, $\mathrm{M}$-cone loss in mice or can lead to severe cone photoreceptor-mediated retinal disease in patients ${ }^{41,50,51}$. Importantly, restoration of CRALBP expression specifically in Müller cells, but not RPE cells, can rescue the sensitivity of CRALBP-deficient cones in the mouse ${ }^{41}$. We can thus raise the hypothesis that the severe decrease of CRALBP levels in Müller cells of aged $\mathrm{Yap}^{+/-}$mice may contribute to the observed cone dysfunction and degeneration. We did not observe any significant decrease in CRALBP levels in Yap CKO retinas (data not shown), which may explain, at least in part, the less severe cone phenotype (PNA staining unaffected) compared to the one observed in $\mathrm{Yap}^{+/-}$mice. This could indicate only structural cone defects in Yap CKO retinas without bona fide degeneration. Besides, the decreased abundance of YAP in $\mathrm{Yap}^{+/-}$mice in other cell types than Müller cells may also explain the different severity of the phenotype. Although we did not detect any major defects in RPE cells in $\mathrm{Yap}^{+/-}$mice, we observed a decreased expression of CRALBP in RPE cells. We therefore cannot exclude the possibility that the RPE is not functioning properly, contributing to cone degeneration. Similarly, the defect in the intermediate vascular plexus observed in $\mathrm{Yap}^{+/-}$mice could also participate to the degenerative phenotype.

We here did not focus our analysis on extraocular tissues. However, ocular phenotypes in a $\mathrm{Yap}^{+/-}$mouse line were recently examined with an emphasis on the cornea and severe corneal pathology was reported ${ }^{52}$. In this mutant mouse, similar to our findings, retinal defects were detected only in aged mice. However, in contrast to our study, the phenotype was very severe, including retinal detachment, defect in the RPE and important loss of photoreceptors. It remains to be addressed whether genetic background differences could explain these phenotypic variabilities between the two $\mathrm{Yap}^{+/-}$mouse lines.

To conclude, we highlighted in this study a novel role for YAP in the maintenance of cone photoreceptors in adult mice, associated with a regulation of Müller cell homeostasis. Overall, our data showing cone degeneration in $\mathrm{Yap}^{+/-}$mice $(i)$ warrant further investigation in patients with YAP heterozygous loss-of-function mutations, regarding their cone-driven vision during aging, and (ii) identify Yap as a novel candidate gene that could account for cone dystrophy in patients with unidentified mutations.

\section{Acknowledgements \\ We are thankful to S. Meilhac, Jeff Wrana, and Seth Blackshaw for mouse lines. We are grateful to S. Lourdel for her help with the maintenance of mouse colonies. This research was supported by grants to M.P. from the FRM \\ (Fondation pour la Recherche Médicale), Association Retina France, Fondation Valentin Haüy, DIM Région Île de France and UNADEV. DGG is a FRM fellow.}

Conflict of interest

The authors declare that they have no conflict of interest.

\section{Publisher's note}

Springer Nature remains neutral with regard to jurisdictional claims in published maps and institutional affiliations.

Supplementary Information accompanies this paper at (https://doi.org/ 10.1038/s41419-020-02860-9).

Received: 23 March 2020 Revised: 29 July 2020 Accepted: 29 July 2020 Published online: 14 August 2020 


\section{References}

1. Zheng, M., Zhang, Z., Zhao, X., Ding, Y. \& Han, H. The Notch signaling pathway in retinal dysplasia and retina vascular homeostasis. J. Genet. Genomics 37, 573-582 (2010)

2. Lad, E. M., Cheshier, S. H. \& Kalani, M. Y. S. Wnt-signaling in retinal development and disease. Stem Cells Dev. 18, 7-16 (2009).

3. Lee, M., Goraya, N., Kim, S. \& Cho, S.-H. Hippo-yap signaling in ocular development and disease. Dev. Dyn. 247, 794-806 (2018).

4. Moon, K. H. \& Kim, J. W. Hippo signaling circuit and divergent tissue growth in mammalian eye. Mol. Cells 41, 257-263 (2018).

5. Edgar, B. A. From cell structure to transcription: Hippo forges a new path. Cell 124, 267-273 (2006)

6. Pan, D. Hippo signaling in organ size control. Genes Dev. 21, 886-897 (2007)

7. Miesfeld, J. B. et al. Yap and Taz regulate retinal pigment epithelial cell fate. Development 142, 3021-3032 (2015).

8. Zhang, H., Deo, M., Thompson, R. C., Uhler, M. D. \& Turner, D. L. Negative regulation of Yap during neuronal differentiation. Dev. Biol. 361, 103-115 (2012).

9. Kim, J. Y. et al. Yap is essential for retinal progenitor cell cycle progression and RPE cell fate acquisition in the developing mouse eye. Dev. Biol. 419, 336-347 (2016).

10. Asaoka, Y., Hata, S., Namae, M., Furutani-Seiki, M. \& Nishina, H. The Hippo pathway controls a switch between retinal progenitor cell proliferation and photoreceptor cell differentiation in zebrafish. PLoS ONE 9, e97365 (2014).

11. Jiang, Q. et al. Yap is required for the development of brain, eyes, and neural crest in zebrafish. Biochem. Biophys. Res. Commun. 384, 114-119 (2009).

12. Fossdal, R. et al. A novel TEAD1 mutation is the causative allele in Sveinsson's chorioretinal atrophy (helicoid peripapillary chorioretinal degeneration). Hum. Mol. Genet. 13, 975-981 (2004).

13. Kitagawa, M. A Sveinsson's chorioretinal atrophy-associated missense mutation in mouse Tead1 affects its interaction with the co-factors YAP and TAZ. Biochem. Biophys. Res. Commun. 361, 1022-1026 (2007).

14. Asthagiri, A. R. et al. Neurofibromatosis type 2. Lancet (Lond., Engl.) 373 1974-1986 (2009).

15. Williamson, K. A. et al. Heterozygous loss-of-function mutations in YAP1 cause both isolated and syndromic optic fissure closure defects. Am. J. Hum. Genet. 94, 295-302 (2014).

16. Oatts, J. T. et al. Novel heterozygous mutation in YAP1 in a family with isolated ocular colobomas. Ophthalmic Genet 38, 281-283 (2017).

17. Hamon, A. et al. Retinal degeneration triggers the activation of YAP/TEAD in reactive Müller. Cells Invest. Opthalmol. Vis. Sci. 58, 1941 (2017).

18. Morin-kensicki, E. M. et al. Defects in yolk sac vasculogenesis, chorioallantoic fusion, and embryonic axis elongation in mice with targeted disruption of Yap65 defects. Mol. Cell Biol. 26, 77-87 (2006).

19. Cao, X., Pfaff, S. L. \& Gage, F. H. YAP regulates neural progenitor cell number via the TEA domain transcription factor. Genes Dev. 22, 3320 (2008).

20. Reginensi, A. et al. Yap- and Cdc42-dependent nephrogenesis and morphogenesis during mouse kidney development. PLoS Genet. 9, e1003380 (2013)

21. Lallemand, Y., Luria, V., Haffner-Krausz, R. \& Lonai, P. Maternally expressed PGKCre transgene as a tool for early and uniform activation of the Cre site-specific recombinase. Transgenic Res. 7, 105-112 (1998).

22. Hamon, A. et al. Linking YAP to Müller Glia quiescence exit in the degenerative retina. Cell Rep. 27, 1712-1725.e6 (2019).

23. Pak, T., Yoo, S., Miranda-Angulo, A. M., Wang, H. \& Blackshaw, S. Rax-CreERT2 knock-in mice: a tool for selective and conditional gene deletion in progenitor cells and radial glia of the retina and hypothalamus. PLOS ONE 9, e90381 (2014).

24. Schindelin, J. et al. Fiji: an open-source platform for biological-image analysis. Nat. Methods 9, 676-682 (2012).

25. Vandesompele, J. et al. Accurate normalization of real-time quantitative RTPCR data by geometric averaging of multiple internal control genes. Genome Biol. 3, 34-1 (2002).

26. Neto, F. et al. YAP and TAZ regulate adherens junction dynamics and endothelial cell distribution during vascular development. elife 7, e31037 (2018).
27. Deng, Y. et al. A reciprocal regulatory loop between TAZNAP and G-protein Gas regulates Schwann cell proliferation and myelination. Nat. Commun. 8, 1-15 (2017).

28. Lai, D., Ho, K. C., Hao, Y. \& Yang, X. Taxol resistance in breast cancer cells is mediated by the hippo pathway component TAZ and its downstream transcriptional targets Cyr61 and CTGF. Cancer Res. 71, 2728-2738 (2011).

29. Yang, R. et al. The Hippo transducer TAZ promotes cell proliferation and tumor formation of glioblastoma cells through EGFR pathway. Oncotarget 7, 36255-36265 (2016).

30. Chen, J. \& Harris, R. C. Interaction of the EGF receptor and the hippo pathway in the diabetic kidney. J. Am. Soc. Nephrol. 27, 1689-1700 (2016).

31. He, C. et al. YAP forms autocrine loops with the ERBB pathway to regulate ovarian cancer initiation and progression. Oncogene 34, 6040-6054 (2015).

32. Close, J. L., Liu, J., Gumuscu, B. \& Reh, T. A. Epidermal growth factor receptor expression regulates proliferation in the postnatal rat retina. Glia 54, 94-104 (2006).

33. Oliveira, C. S., Rigon, A. P., Leal, R. B. \& Rossi, F. M. The activation of ERK1/2 and p38 mitogen-activated protein kinases is dynamically regulated in the developing rat visual system. Int. J. Dev. Neurosci. 26, 355-362 (2008).

34. Ornelas, I. M., Silva, T. M., Fragel-Madeira, L. \& Ventura, A. L. M. Inhibition of PI3K/Akt pathway impairs G2/M transition of cell cycle in late developing progenitors of the avian embryo retina. PLOS ONE 8, e53517 (2013).

35. Zhang, S. S. M. et al. STAT3 activation in response to growth factors or cytokines participates in retina precursor proliferation. Exp. Eye Res. 81, 103-115 (2005).

36. Yu, F. X., Zhao, B. \& Guan, K. L. Hippo pathway in organ size control, tissue homeostasis, and cancer. Cell 163, 811-828 (2015).

37. Fu, V., Plouffe, S. W. \& Guan, K.-L. The Hippo pathway in organ development, homeostasis, and regeneration. Curr. Opin. Cell Biol. 49, 99-107 (2017).

38. Zhang, $\mathrm{H}$. et al. The degeneration and apoptosis patterns of cone photoreceptors in rd11 mice. J. Ophthalmol. 2017, 9721362 (2017)

39. Reichenbach, A. \& Bringmann, A. New functions of Müller cells. Glia https://doi. org/10.1002/glia.22477 (2013).

40. Mata, N. L., Radu, R. A., Clemmons, R. C. \& Travis, G. H. Isomerization and oxidation of vitamin a in cone-dominant retinas: a novel pathway for visualpigment regeneration in daylight. Neuron 36, 69-80 (2002).

41. Xue, Y. et al. CRALBP supports the mammalian retinal visual cycle and cone vision. J. Clin. Invest. 125, 727-738 (2015).

42. Wang, J. \& Kefalov, V. J. The cone-specific visual cycle. Prog. Retin Eye Res. 30 115-128 (2011).

43. Coorey, N. J., Shen, W., Chung, S. H., Zhu, L. \& Gillies, M. C. The role of glia in retinal vascular disease. Clin. Exp. Optom. 95, 266-281 (2012)

44. Kim, J. et al. YAP/TAZ regulates sprouting angiogenesis and vascular barrier maturation. J. Clin. Invest. 127, 3441-3461 (2017).

45. Finch-Edmondson, M. L. et al. TAZ protein accumulation is negatively regulated by YAP abundance in mammalian cells. J. Biol. Chem. 290, 27928-27938 (2015).

46. Cabochette, $P$. et al. YAP controls retinal stem cell DNA replication timing and genomic stability. elife 4, e08488 (2015).

47. Dupont, S. et al. Role of YAP/TAZ in mechanotransduction. Nature 474 179-183 (2011).

48. Shen, W. et al. Conditional Müller cell ablation causes independent neuronal and vascular pathologies in a novel transgenic model. J. Neurosci. 32 , 15715-15727 (2012)

49. Byrne, L. C. et al. AAV-mediated, optogenetic ablation of Müller Glia leads to structural and functional changes in the mouse retina. PLOS ONE 8, e76075 (2013).

50. Fleisch, V. C., Schonthaler, H. B., von Lintig, J. \& Neuhauss, S. C. F. Subfunctionalization of a retinoid-binding protein provides evidence for two parallel visual cycles in the cone-dominant zebrafish retina. J. Neurosci. $\mathbf{2 8}$ 8208-8216 (2008).

51. Maw, M. A. et al. Mutation of the gene encoding cellular retinaldehydebinding protein in autosomal recessive retinitis pigmentosa. Nat. Genet. 17, 198-200 (1997).

52. Kim, S. et al. Ocular phenotypic consequences of a single copy deletion of the Yap1 gene (Yap1+/-) in mice. Mol. Vis. 25, 129-142 (2019). 